\title{
The Forcing Relation for Horseshoe Braid Types
}

\author{
André de Carvalho and Toby Hall
}

\section{CONTENTS}

1. Introduction

2. Background

3. The Conjecture

4. Evidence for the Conjecture

5. Appendix: Proof of Lemma 3.3

References
2000 AMS Subject Classification: Primary 37E15, 37E30; Secondary 37B10, 57M25

Keywords: Horseshoe periodic orbits, braid forcing
This paper presents evidence for a conjecture concerning the structure of the set of braid types of periodic orbits of Smale's horseshoe map, partially ordered by Boyland's forcing order. The braid types are partitioned into totally ordered subsets, which are defined by parsing the symbolic code of a periodic orbit into two segments, the prefix and the decoration: The set of braid types of orbits with each given decoration is totally ordered, the order being given by the unimodal order on symbol sequences. The conjecture is supported by computer experiment, by proofs of special cases, and by intuitive argument in terms of pruning theory.

\section{INTRODUCTION}

This paper presents strong evidence for a conjecture concerning the order in which periodic orbits can appear in the creation of Smale's horseshoe [Smale 67]. Since any $C^{1+\epsilon}$ surface diffeomorphism with positive topological entropy has horseshoes in some iterate [Katok 80], an understanding of the mechanism of horseshoe creation provides insight into the mechanism of transitions to positive entropy for general surface diffeomorphisms. As such, this problem has received a good deal of attention over the last 20 years.

The conjecture is stated in terms of Boyland's forcing order [Boyland 84] on the set of braid types of periodic orbits of the horseshoe (see Section 2.1). Loosely stated, the periodic orbit $P$ forces the periodic orbit $Q$ if an orbit of the same type as $Q$ must be present in the dynamics of any homeomorphism which has an orbit of the same type as $P$.

The conjecture is based on a parsing of the symbolic code of each horseshoe periodic orbit into two segments, the prefix and the decoration (see Section 3.1). Writing $\mathcal{D}_{w}$ for the family of all periodic orbits with a given decoration $w$, the main claims of the conjecture are:

(a) Each family $\mathcal{D}_{w}$ is totally ordered by the forcing order, and this order coincides with the unimodal order on symbol sequences.

(c) A K Peters, Ltd. 1058-6458/2001 $\$ 0.50$ per page Experimental Mathematics 11:2, page 271 
(b) All of the orbits in each given family $\mathcal{D}_{w}$ have the same topological train track type (see Section 3.3).

(c) The forcing order between different families $\mathcal{D}_{w}$ can be understood in terms of homoclinic orbits associated to the families.

As will be discussed in more detail below, Thurston's classification theorem for isotopy classes of surface homeomorphisms [Thurston 88], used in conjunction with a train track algorithm such as that of Bestvina and Handel [Bestvina and Handel 95], makes it theoretically possible - if practically very time consuming - to decide whether and how two orbits are related by the forcing order. Despite this attractive theoretical background, it has proved very difficult to describe the global structure of the forcing order.

The conjecture presented here makes the calculation of the order nearly trivial within families: simply compare their symbol sequences using the unimodal order. Moreover, it gives a global description of how the set of horseshoe braid types is organized, information that could not be obtained by comparing braids pairwise.

As the terminology suggests, this work has connections with braid theory. In Section 2.2, it is explained how the conjecture, if proved, would also provide an efficient partial solution to the conjugacy problem for cyclic unimodal permutation braids.

Some well-established background to the problem is presented in Section 2: braid types, Boyland's forcing order, Thurston's classification theorem for surface homeomorphisms, and the notion of the height of a periodic orbit of the horseshoe. Section 3 is more directly concerned with the conjecture: it describes how symbolic codes are parsed into prefix and decoration, summarizes some well-known results arising from the symmetry of the horseshoe and its inverse, and introduces the notion of topological train track types. The main conjecture is stated in Section 3.4. Section 4 is concerned with evidence for the conjecture: proofs of special cases, computational evidence, and intuitive arguments.

\section{BACKGROUND}

\subsection{Braid Types and the Thurston Classification}

Braid types were introduced by Boyland [Boyland 84] as an algebraic specification of periodic orbits of surface homeomorphisms: the braid type of a periodic orbit $P$ of a surface homeomorphism $f: S \rightarrow S$ is essentially the isotopy class of $f$ relative to $P$. For the sake of simplicity, the definition given here is restricted to orientationpreserving homeomorphisms of the disk, which is the case of interest in this paper. The definition makes sense for arbitrary invariant sets, not just for periodic orbits; in Section 2.2, it is extended to certain homoclinic orbits.

Definitions 2.1. Let $D^{2}$ be the unit disk in the plane and let $f: D^{2} \rightarrow D^{2}$ and $g: D^{2} \rightarrow D^{2}$ be orientationpreserving homeomorphisms having periodic orbits $P$ and $Q$, respectively. If $P$ (respectively $Q$ ) lies on $\partial D^{2}$, then extend $f$ (respectively $g$ ) as a homeomorphism over an exterior collar of $D^{2}$ (and use the same notation $D^{2}$ to denote the collared disk). Then $(P, f)$ and $(Q, g)$ have the same braid type if there is an orientation-preserving homeomorphism $h: D^{2} \rightarrow D^{2}$ with $h(P)=Q$ such that $f$ and $h^{-1} \circ g \circ h$ are isotopic relative to $P$. This defines an equivalence relation on the set of all pairs $(P, f)$ : the equivalence class of $(P, f)$ is denoted $\operatorname{bt}(P, f)$; the braid type of the periodic orbit $P$ of $f$.

The set of all braid types of periodic orbits of orientation-preserving homeomorphisms of the disk is denoted BT. Clearly, two periodic orbits with the same braid type must have the same period: the set of all braid types of period $n$ orbits of orientation-preserving homeomorphisms of the disk is denoted $\mathrm{BT}_{n}$. Given an orientation-preserving homeomorphism $f: D^{2} \rightarrow D^{2}$, write

$$
\operatorname{bt}(f)=\{\operatorname{bt}(P, f): P \text { is a periodic orbit of } f\} .
$$

The term braid type is appropriate because the group of isotopy classes of orientation-preserving homeomorphisms of the $n$-punctured disk is isomorphic to the $n$ braid group $B_{n}$ modulo its centre. This isomorphism induces a canonical bijection between $\mathrm{BT}_{n}$ and the set of conjugacy classes in $B_{n} / Z\left(B_{n}\right)$, which provides a convenient way of representing braid types.

One of the main endeavours in this area is to understand which braid types must necessarily coexist with a given braid type; this is formalized by Boyland's forcing order on BT [Boyland 84].

Definition 2.2. The forcing order $\leq$ on BT is defined as follows: If $\beta, \gamma \in \mathrm{BT}$, then $\gamma \leq \beta$ if and only if for all homeomorphisms $f: D^{2} \rightarrow D^{2}, \beta \in \operatorname{bt}(f) \Longrightarrow \gamma \in$ $\operatorname{bt}(f)$. If $\gamma \leq \beta$, then one says that $\beta$ forces $\gamma$.

It is obvious that $\leq$ is reflexive and transitive; its antisymmetry was proved by Boyland [Boyland 94]:

Theorem 2.3. (Boyland.) $\leq$ is a partial order on BT. 
The main tool for understanding the structure of this partially ordered set is Thurston's classification theorem for isotopy classes of surface homeomorphisms [Thurston 88]. This will be stated here for orientation-preserving homeomorphisms of the punctured disk only.

Definitions 2.4. Let $A$ be a finite subset of $D^{2} \backslash \partial D^{2}$.

A simple closed curve in $D^{2} \backslash A$ is essential if it bounds a disk containing more than one but fewer than all of the points of $A$.

An isotopy class $\alpha$ of orientation-preserving homeomorphisms of $\left(D^{2}, A\right)$ is reducible if there exists an element $f:\left(D^{2}, A\right) \rightarrow\left(D^{2}, A\right)$ of $\alpha$ and a finite reducing collection of pairwise disjoint and nonhomotopic essential simple closed curves which are permuted by $f$.

A pseudo-Anosov homeomorphism $\phi:\left(D^{2}, A\right) \rightarrow$ $\left(D^{2}, A\right)$ is one for which there exists a number $\lambda>1$ and a pair $\left(\mathcal{F}_{s}, \mu_{s}\right),\left(\mathcal{F}_{u}, \mu_{u}\right)$ of transverse measured foliations of $D^{2}$ such that $\phi\left(\mathcal{F}_{s}, \mu_{s}\right)=\left(\mathcal{F}_{s}, \lambda^{-1} \mu_{s}\right)$ and $\phi\left(\mathcal{F}_{u}, \mu_{u}\right)=\left(\mathcal{F}_{u}, \lambda \mu_{u}\right)$. The foliations can have a finite number of singular points where they each have $p \neq 2$ prongs, but 1-pronged singularities can only occur at points of $A$ and on $\partial D^{2}$.

The idea is that if an isotopy class $\alpha$ is reducible, then one can cut along the reducing curves and study the action of $\alpha$ on the simpler pieces into which the punctured disk is divided. Thurston's classification theorem provides a canonical representative of each irreducible isotopy class.

Theorem 2.5. (Thurston.) Let $\alpha$ be an irreducible isotopy class of orientation-preserving homeomorphisms of $\left(D^{2}, A\right)$. Then exactly one of the following occurs:

(i) $\alpha$ contains a finite order homeomorphism $\phi$ (i.e., $\phi^{n}=$ id for some $n>0$ : this implies that $\phi$ is conjugate to a rigid rotation of $D^{2}$ ).

(ii) a contains a pseudo-Anosov homeomorphism $\phi$.

The irreducible isotopy class $\alpha$ is said to be finite order or pseudo-Anosov according as it contains a finite order or pseudo-Anosov homeomorphism. The detailed properties of pseudo-Anosov homeomorphisms will not be used here: the important property from a dynamical point of view is that they have minimal dynamics within their isotopy class. In particular [Fathi et. al. 79, Hall 91],

Theorem 2.6. Let $\phi$ be a pseudo-Anosov homeomorphism of $\left(D^{2}, A\right)$ and let $f:\left(D^{2}, A\right) \rightarrow\left(D^{2}, A\right)$ be isotopic to $\phi$.
Then

(i) $h(\phi) \leq h(f)$.

(ii) $\operatorname{bt}(\phi) \subseteq \operatorname{bt}(f)$.

Here $h(f)$ denotes the topological entropy of $f$ [Adler et al. 65]. Property (ii) is the one which is useful in understanding the structure of $(B T, \leq)$. Since the Thurston classification is invariant under conjugation, each braid type $\beta$ can be classified as reducible, finite order, or pseudo-Anosov, according to the isotopy class of $f:\left(D^{2}, P\right) \rightarrow\left(D^{2}, P\right)$, where $\operatorname{bt}(P, f)=\beta$. A finite order braid type $\beta$ can be realised by a rigid rotation, and hence only forces itself and the braid type of a fixed point. If $\beta$ is a pseudo-Anosov braid type, then let $\phi_{\beta}:\left(D^{2}, P\right) \rightarrow\left(D^{2}, P\right)$ be a representative pseudoAnosov homeomorphism.

Corollary 2.7. Let $\beta \in \mathrm{BT}$ be pseudo-Anosov. Then

$$
\{\gamma \in \mathrm{BT}: \gamma \leq \beta\}=\operatorname{bt}\left(\phi_{\beta}\right) .
$$

Several algorithms (e.g., [Bestvina and Handel 95, Benardete et al. 93, Benardete et al. 95, Franks and Misiurewicz 93, Los 93, de Carvalho and Hall 01]) have been presented which, given as input an isotopy class of orientation-preserving homeomorphisms of $\left(D^{2}, A\right)$, determine whether it is reducible, finite order, or pseudoAnosov, and provide a set of reducing curves or an explicit construction of a pseudo-Anosov homeomorphism in the isotopy class in the reducible and pseudo-Anosov cases, respectively. In principle, these make it possible to calculate whether or not $\gamma \leq \beta$ for any $\beta, \gamma \in \mathrm{BT}$. If $\beta$ is pseudo-Anosov, the output of the algorithm is an invariant train track (see Section 3.3) which enables one to enumerate all of the periodic orbits of $\phi_{\beta}$ of the appropriate period, and test each in turn to determine whether or not its braid type is $\gamma$. In practice, this takes a very long time; the tests involve solving the conjugacy problem in the braid group. Moreover, the ability to carry out such local calculations does not provide any insight into the global structure of $(\mathrm{BT}, \leq)$.

\subsection{The Height of a Periodic Orbit of the Horseshoe}

It is assumed that the reader is familiar with Smale's horseshoe map [Smale 67], with the standard procedure for introducing symbolic dynamics on its nonwandering set, and with the unimodal order on the resulting symbol space. This material can be found in many standard texts on dynamical systems (e.g., [Devaney 89]). In 


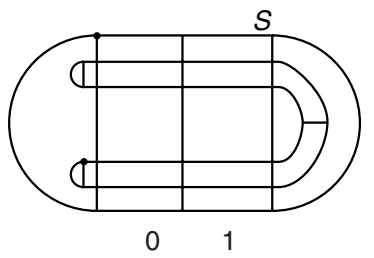

FIGURE 1. Symbolic dynamics for the horseshoe.

this paper, symbolic dynamics in $\Sigma_{2}=\{0,1\}^{\mathbb{Z}}$ will be used on a standard model $F: D^{2} \rightarrow D^{2}$ of the horseshoe as depicted in Figure 1. The symbol $F$ will always denote the horseshoe map, and $k: \Lambda \rightarrow \Sigma_{2}$ will denote the itinerary homeomorphism which conjugates the shift map $\sigma: \Sigma_{2} \rightarrow \Sigma_{2}$ to $\left.F\right|_{\Lambda}: \Lambda \rightarrow \Lambda$, where $\Lambda$ is the set of points whose (past and future) orbits lie entirely in the square $S$. If $x \in \Lambda$, then $k(x) \in \Sigma_{2}$ is called the itinerary of $x$. The definitions and results summarized in this section can be found in [Hall 94a].

A period $n$ orbit $P$ of $F$ will be described by its code $c_{P} \in\{0,1\}^{n}$, which is given by the first $n$ symbols of the itinerary of its rightmost point $p$ : thus

$$
k(p)=\overline{c_{P}} \cdot \overline{c_{P}}
$$

(here and throughout, an overbar denotes infinite repetition, and a $\cdot$ appears before the zeroth symbol of an element of $\Sigma_{2}$ ). For example, the period 5 periodic orbit which contains the point with itinerary $\overline{01001}$ has code 10010. Put another way, the word $c_{P} \in\{0,1\}^{n}$ is the code of a period $n$ orbit of the horseshoe if and only if the semi-infinite sequence $\overline{c_{P}}$ is strictly greater than $\sigma^{i}\left(\overline{c_{P}}\right)$ in the unimodal order for $1 \leq i<n$. This paper is also concerned with homoclinic orbits $H$ to the fixed point with code 0: such an orbit will be described by its core $c_{H}$, which is the nonzero segment in the itineraries of its points (so, for example, the homoclinic orbit which contains the point with itinerary $\overline{0} 11 \cdot 001 \overline{0}$ has core 11001). In the remainder of the paper, "homoclinic" will always mean homoclinic to this fixed point. The braid type bt $(P, F)$ of a periodic orbit $P$ of the horseshoe will be denoted bt $(P)$; and the notation $P \geq Q$ will be used as an abbreviation for $\operatorname{bt}(P) \geq \operatorname{bt}(Q)$. Similarly, it is possible to define the homoclinic braid type $\operatorname{hbt}(H)$ of a homoclinic orbit of the horseshoe: two homoclinic orbits $H$ and $H^{\prime}$ of homeomorphisms $f$ and $g$ have the same homoclinic braid type if there is an orientation-preserving homeomorphism $h: D^{2} \rightarrow D^{2}$ with $h(H)=H^{\prime}$ such that $f$ is isotopic to $h^{-1} \circ g \circ h$ relative to $H$. The notation $H \geq H^{\prime}$ means that every homeomorphism of the disk which has a homoclinic orbit of homoclinic braid type
$\operatorname{hbt}(H)$ also has one of homoclinic braid type $\operatorname{hbt}\left(H^{\prime}\right)$. Let $\mathrm{HS}=\mathrm{bt}(F)$ denote the set of braid types of periodic orbits of the horseshoe, and HHS the set of homoclinic braid types of the horseshoe.

The aim of this paper is to describe the structure of the partially ordered set $(\mathrm{HS}, \leq)$ : this gives information about the way in which periodic orbits are created in parameterized families of homeomorphisms leading to the creation of a horseshoe. According to Conjecture 3.10, this problem reduces to that of understanding the relation $\leq$ on HHS: the set of nonfinite order elements of HS can be partitioned into totally ordered families on which the order is well understood; and there is a bijection between HHS and the set of families, such that the ordering of braid types in two different families can be easily determined provided it is known how the corresponding homoclinic braid types are related by $\leq$. Note that if $\mathrm{HS}^{*}=\mathrm{HS} \cup \mathrm{HHS}$, then $\leq$ extends in a natural way to a relation on HS* A consequence of Conjecture 3.10 is that this extended relation is also a partial order.

It is well known that two periodic orbits $P$ and $Q$ of the horseshoe whose codes $c_{P}$ and $c_{Q}$ differ only in their final symbol have the same braid type. Thus, for example, the two orbits with codes 10010 and 10011 have the same braid type; the code of either one of these orbits is often written $c_{P}=1001_{1}^{0}$ to reflect the fact that the distinction between the two is unimportant in so far as $(\mathrm{HS}, \leq)$ is concerned. However, this is not the only way in which two horseshoe orbits can have the same braid type: the conjecture also gives necessary and sufficient conditions (which may or may not be verifiable in practice) for two periodic orbits to have the same braid type. In another language, this provides an efficient partial solution to the conjugacy problem for cyclic unimodal permutation braids.

The remainder of this section is devoted to describing the height $q(P)$ of a horseshoe periodic orbit $P$ of period greater than 1 . The height is a invariant of braid type with values in $(0,1 / 2] \cap \mathbb{Q}$ which plays a central role in the conjecture. The description is rather complicated at first sight; a program for computing heights of horseshoe orbits can be found at [Hall 02], and motivation for the definition is given in [Hall 94a].

Algorithm 2.8. Let $P$ be a horseshoe periodic orbit which is not a fixed point, with code $c=c_{P}$. If the semi-infinite sequence $\bar{c}$ does not contain the word 010, then change the final symbol of $c$ from 1 to 0 . Now write

$$
\bar{c}=10^{\kappa_{1}} 1^{\mu_{1}} 0^{\kappa_{2}} 1^{\mu_{2}} \ldots,
$$


where each $\kappa_{i} \geq 0$, each $\mu_{i}$ is either 1 or 2 , and $\mu_{i}=1$ only if $\kappa_{i+1}>0$ (thus $\kappa_{i}$ and $\mu_{i}$ are uniquely determined by $c$ ). For each $r \geq 1$, define

$$
I_{r}(c)=\left(\frac{r}{2 r+\sum_{i=1}^{r} \kappa_{i}}, \frac{r}{(2 r-1)+\sum_{i=1}^{r} \kappa_{i}}\right],
$$

and let $s \geq 1$ be the least integer such that either $\mu_{s}=1$ or $\bigcap_{i=1}^{s+1} I_{i}(c)=\emptyset$. Write $\bigcap_{i=1}^{s} I_{i}(c)=(x, y]$. Then

$q(P)= \begin{cases}x & \text { if } \mu_{s}=2 \text { and } w<z \\ & \text { for all } w \in I_{s+1}(c) \text { and } z \in \bigcap_{i=1}^{s} I_{i}(c) \\ y & \text { if } \mu_{s}=1, \text { or } \mu_{s}=2 \text { and } w>z \\ & \text { for all } w \in I_{s+1}(c) \text { and } z \in \bigcap_{i=1}^{s} I_{i}(c) .\end{cases}$

Notice that some $\mu_{i}$ is equal to 1 (since $\bar{c}$ contains the word 010), and hence the algorithm terminates.

Example 2.9. Let $P$ be the period 17 orbit with code 10011011001011010. Then $\kappa_{1}=2, \mu_{1}=2, \kappa_{2}=1$, $\mu_{2}=2, \kappa_{3}=2$, and $\mu_{3}=1$. Thus $I_{1}=(1 / 4,1 / 3]$, $I_{2}=(2 / 7,2 / 6]$, and $I_{3}=(3 / 11,3 / 10]$. Since $\mu_{3}=1$, the algorithm terminates with $\bigcap_{i=1}^{3} I_{i}=(2 / 7,3 / 10]$, and hence $q(P)=3 / 10$.

The following theorem is a summary of the relevant results from [Hall 94a]. Given $q=m / n \in(0,1 / 2]$ in lowest terms, define $c_{q} \in\{0,1\}^{n+1}$ by

$$
c_{q}=10^{\kappa_{1}} 1^{2} 0^{\kappa_{2}} 1^{2} \ldots 1^{2} 0^{\kappa_{m}} 1,
$$

where

$$
\kappa_{i}= \begin{cases}\lfloor n / m\rfloor-1 & \text { if } i=1 \\ \lfloor i n / m\rfloor-\lfloor(i-1) n / m\rfloor-2 & \text { if } 2 \leq i \leq m\end{cases}
$$

(here $\lfloor x\rfloor$ denotes the greatest integer which does not exceed $x$ ). The words $c_{q}$ are palindromic: that is, $\kappa_{i}=$ $\kappa_{m+1-i}$ for all $i$.

Theorem 2.10. Let $P$ and $Q$ be periodic orbits of the horseshoe.

(i) If $P$ and $Q$ have the same braid type, then $q(P)=$ $q(Q)$.

(ii) If $P \geq Q$, then $q(P) \leq q(Q)$.

(iii) Let $q(P)=m / n$ in lowest terms. Then $P$ has period $n$ if and only if it has finite order braid type: in this case, $F$ is isotopic rel. $P$ to a rigid rotation through $2 \pi m / n$. Otherwise, the period of $P$ is at least $n+2$, and $c_{P}$ starts with the word $c_{m / n}$.
In fact, $q(P)$ has a dynamical interpretation: it is the lefthand endpoint of the rotation interval of $P$. The definition of the height can be extended to all sequences which contain the word 010 ; this extension will be used in the statement of Lemma 3.3.

Definition 2.11. Let $\mathcal{C}$ denote the subset of $\{0,1\}^{\mathbb{N}}$ consisting of sequences which contain the word 010 . The height $q(c)$ of an element $c \in \mathcal{C}$ is defined by $q(c)=1 / 2$ if $c$ does not begin $c=10 \ldots$, and by the above algorithm, otherwise.

The function $q: \mathcal{C} \rightarrow(0,1 / 2] \cap \mathbb{Q}$ is decreasing with respect to the unimodal order on $\Sigma_{2}$ and the usual order on $\mathbb{Q}$.

\section{THE CONJECTURE}

\subsection{Prefix and Decoration}

Let $P$ be a periodic orbit of the horseshoe. If $P$ is a fixed point, then clearly it forces only the fixed point braid type. If $P$ is not a fixed point, then it has a well-defined height $q(P)=m / n \in(0,1 / 2]$, written in lowest terms. If $P$ has period $n$, then, by Theorem 2.10 (iii), it has finite order braid type with rotation number $m / n$. In fact, Holmes and Williams showed [Holmes and Williams 85] that for each $m / n \in(0,1 / 2)$, there is exactly one pair of periodic orbits with this finite order braid type $\alpha_{m / n}$ : one has code given by the first $n$ symbols of $c_{m / n}$, and the other has the same code with the final 1 changed to a 0 . There is just one periodic orbit of braid type $\alpha_{1 / 2}$, namely the period 2 orbit with code 10 .

These orbits will be ignored in the remainder of the paper; it is obvious what they force, and it is known which other orbits force them $\left(P \geq \alpha_{q}\right.$ if and only if $q$ is in the rotation interval of $P$ by a theorem of Boyland [Boyland $92]$, and an algorithm for computing rotation intervals of horseshoe orbits is given in [Hall 94a]).

The code of any other periodic orbit $P$ can be written in the form

$$
c_{P}=c_{q(P)} v
$$

for some word $v$ of length at least 1, by Theorem $2.10 \mathrm{c}$ ).

Definition 3.1. Let $P$ be a period $N$ orbit of the horseshoe which is not of finite order braid type, with height $q=q(P)=m / n$. The prefix of $P$ is the word $c_{q}$. The decoration of $P$ is defined to be $\star$ if $N=n+2$, and to be the element $w$ of $\{0,1\}^{N-n-3}$ such that

$$
c_{P}=c_{q}{ }_{1}^{0} w_{1}^{0}
$$


if $N \geq n+3$. The notation $P=P_{q}^{w}$ means that $P$ is a periodic orbit of height $q$ and decoration $w$. The choice of 0 or 1 for the final symbol of $c_{P}$ is almost always unimportant, but the choice of the symbol before the decoration can be significant. Where it is, the periodic orbits with codes $c_{q} 0 w_{1}^{0}$ and $c_{q} 1 w_{1}^{0}$ will be denoted $P_{q}^{w}(0)$ and $P_{q}^{w}(1)$, respectively.

Example 3.2. Let $P$ be the period 17 orbit with code $c_{P}=$ 10011011001011010. Then $q(P)=3 / 10$ as shown in the previous example. Hence, $P$ has prefix $10011011001=$ $c_{3 / 10}$ and decoration 1101 . Thus $P=P_{3 / 10}^{1101}$. Where the distinction is important, $P$ could be denoted $P_{3 / 10}^{1101}(0)$.

Hence, every periodic orbit which is not of finite order braid type can be written as $P_{q}^{w}$, where $q=q(P)$ and $w$ is the decoration of $P$. This means precisely that the orbit has one of the four codes $c_{q 1}^{0} w_{1}^{0}$, unless $w=\star$, in which case it has one of the two codes $c_{q 1}^{0}$. It is convenient to extend this notation to homoclinic orbits, writing $P_{0}^{w}$ for the homoclinic orbits with core $1_{1}^{0} w_{1}^{0} 1$ (and $P_{0}^{\star}$ for the homoclinic orbits with core $1_{1}^{0} 1$ ). This description omits only those homoclinic orbits whose core has length 2 or less: these are precisely the homoclinic orbits of translation homoclinic braid type, which force no other homoclinic or periodic braid types. In the next subsection, we show that the four homoclinic orbits described by the symbol $P_{0}^{w}$ all have the same homoclinic braid type. Likewise, it is shown in [de Carvalho and Hall 02a] that all of the (four or fewer) periodic orbits described by the symbol $P_{q}^{w}$ have the same braid type.

Only certain heights $q$ are compatible with each given decoration. Since the code $c_{P}$ of a period $N$ orbit $P$ corresponds to its rightmost point, it must be maximal: that is, $\sigma^{i}\left(\overline{c_{P}}\right)$ must be strictly less than $\overline{c_{P}}$ in the unimodal order for all integers $i$ with $1 \leq i<N$. The set of heights compatible with a given decoration $w$ is described by the following lemma. Although this lemma plays a central role in the paper, its proof depends on technical results from [Hall 94a], and as such has been relegated to an appendix.

Lemma 3.3. Let $w$ be a decoration, and define $q_{w} \in$ $(0,1 / 2] \cap \mathbb{Q}$ by $q_{\star}=1 / 2$ and

$$
q_{w}=\min _{0 \leq i \leq k+2} q\left(\sigma^{i}(\overline{10 w 0})\right)
$$

if $w \in\{0,1\}^{k}$. Then each of the four codes $c_{q 1}^{0} w_{1}^{0}$ (or each of the two codes $c_{q 1}^{0}$ when $w=\star$ ) is maximal of height $q$ when $0<q<q_{w}$, and none is maximal of height $q$ when $q_{w}<q \leq 1 / 2$.

Example 3.4. When $q=q_{w}$, some, none, or all of the codes $c_{q}{ }_{1}^{0} w_{1}^{0}$ may be maximal. For example, let $w=\star$ : then $q_{w}=1 / 2$. Since $c_{1 / 2}=101$, the two codes concerned are 1011 (which is maximal), and 1010 (which is not). Thus the periodic orbit $P$ with code $c_{P}=1011$ is the only periodic orbit with height $1 / 2$ and decoration $\star$. If $w=0$, then $q_{w}=1 / 4$, and $c_{1 / 4}=10001$. Of the four codes 10001101, 10001001, 10001101, and 10001000, only the last is not maximal. If $w=110$ then $q_{w}=1 / 3$, and $c_{1 / 3}=1001$. All of the four codes 100111101, 100101101, 100111100, and 100101100 are maximal. On the other hand, if $w=10011010$, then $q_{w}=1 / 3$, but none of the four codes 10011100110101, 10010100110101, 10011100110100, and 10010100110100 is maximal.

The point of the phrase "of height $q$ " in the statement of this lemma is that $c_{q 1}^{0} w_{1}^{0}$ may be a maximal code for $q>q_{w}$, but with a different height (and hence a different decoration). For example, if $w=\emptyset$ then $q_{w}=1 / 3$. Let $q=2 / 5>q_{w}$ : then $c_{q} 1_{1}^{0}=1011011_{1}^{0}$ is maximal, but has height $3 / 8$, and is the code of a finite order orbit (and hence has no decoration).

A program for computing $q_{w}$ can be found at [Hall 02]. Note that $q_{w}$ is the height of the periodic orbit containing the point of itinerary $\overline{10 w 0}$ - this orbit need not, of course, have code $10 w 0$.

For each decoration $w$, let $\mathcal{D}_{w}$ denote the set of all periodic and homoclinic orbits of the horseshoe with decoration $w$ : thus

$$
\mathcal{D}_{w}=\left\{P_{q}^{w}: 0 \leq q<=q_{w}\right\}
$$

where the symbol $<=$ denotes that $q=q_{w}$ is possible for some decorations, but not for others.

Convention 3.5. In what follows, whenever the symbol $P_{q}^{w}$ is used it is assumed that the height $q$ is compatible with the decoration $w$.

Summarizing the results of this section: the union of the sets $\mathcal{D}_{w}$ is precisely the set of all periodic and homoclinic orbits of the horseshoe less the periodic orbits of finite order braid type and the homoclinic orbits of translation homoclinic braid type. One of the main claims of Conjecture 3.10 is that each $\mathcal{D}_{w}$ is totally ordered by the forcing order $\leq$. 


\subsection{Reversing Horseshoe Codes}

Let $P$ be a period $N$ orbit of the horseshoe with code $c_{P}$, and let $\widehat{c_{P}}$ denote the reverse of the code $c_{P}$. Let $\widehat{P}$ be the period $N$ orbit containing the point of itinerary $\overline{\widehat{c_{P}}} \cdot \overline{\widehat{c_{P}}}$. The code $c_{\widehat{P}}$ is therefore a cyclic permutation of $\widehat{c_{P}}$.

Example 3.6. Let $P$ be the periodic orbit with code 1001011. Then $\widehat{P}$ is the periodic orbit which contains the point with itinerary $\overline{1101001} \cdot \overline{1101001}$ : thus it has code 1001110 .

Let $\beta_{P}$ be the $N$-braid representing the period $N$ orbit $P$ which is obtained from the natural suspension of the horseshoe (see [Hall 94a] for more details): thus bt $(P)$ is represented by the conjugacy class of $\beta_{P} Z\left(B_{N}\right)$ in $B_{N} / Z\left(B_{N}\right)$. Given $\beta \in B_{N}$, let $\widehat{\beta}$ be the element of $B_{N}$ obtained by writing $\beta$ in terms of the standard Artin generators $\sigma_{i}$ of $B_{N}$, and then reversing the order of these generators (this is a well-defined operation, since the relations between the Artin generators are symmetric under order reversal). It is a well-known consequence of the symmetry of the horseshoe map $F$ and its inverse that $\beta_{\widehat{P}}=\widehat{\beta_{P}^{\prime}}$, where $\beta_{P}^{\prime}$ is the braid obtained by looking at the natural suspension of $P$ from the right rather than from the front of the horseshoe (and is thus conjugate to $\left.\beta_{P}\right)$. That is,

Lemma 3.7. If $\mathrm{bt}(P)$ is represented by the braid $\beta_{P}$, then $\operatorname{bt}(\widehat{P})$ is represented by the braid $\widehat{\beta_{P}}$. In particular, if two horseshoe orbits $P$ and $Q$ have the same braid type, then $\widehat{P}$ and $\widehat{Q}$ have the same braid type.

Because the braids $\beta$ and $\widehat{\beta}$ close to the same knot, it is in general difficult to determine whether or not they are conjugate. In particular, traditional dynamical invariants cannot distinguish between them by the following lemma, which follows easily from the previous one and the uniqueness of pseudo-Anosov representatives of pseudo-Anosov isotopy classes.

Lemma 3.8. Suppose that $P$ is a horseshoe periodic orbit of pseudo-Anosov braid type, and let $\phi_{P}$ be a pseudo-Anosov homeomorphism in the isotopy class of $F:\left(D^{2}, P\right) \rightarrow\left(D^{2}, P\right)$. Then $\widehat{P}$ also has pseudo-Anosov braid type, and

$$
\phi_{\widehat{P}}=h^{-1} \circ \phi_{P}^{-1} \circ h
$$

for some orientation-reversing homeomorphism $h:\left(D^{2}, \widehat{P}\right) \rightarrow\left(D^{2}, P\right)$.
It seems unlikely that $P$ and $\widehat{P}$ always have the same braid type for any horseshoe orbit $P$, although the authors know of no example for which these braid types are different (there are none up to period 9). They would be grateful to hear from anyone who can resolve this problem. (In the language of braid theory, the question is whether or not there exist cyclic unimodal permutation braids $\beta$ which are not conjugate to $\widehat{\beta}$.) It is proved in [Hall 94a] that $q(P)=q(\widehat{P})$ for all horseshoe orbits $P$; this fact will be used in Section 3.4.

The fact, mentioned earlier, that two periodic orbits $P$ and $Q$ whose codes differ only in their final symbol have the same braid type, follows from the observation that their natural braid representatives $\beta_{P}$ and $\beta_{Q}$ are equal. The same observation shows that for any decoration $w \neq$ $\star$, the two homoclinic orbits with cores $11 w_{1}^{0} 1$ have the same homoclinic braid type, as do the two with cores $10 w_{1}^{0} 1$. By the same reversal construction as for periodic orbits, the fact that the orbits with cores $11 \widehat{w}_{1}^{0} 1$ have the same homoclinic braid type means that the orbits with cores $1_{1}^{0} w 11$ have the same homoclinic braid type. Hence, all four homoclinic orbits with cores $1_{1}^{0} w_{1}^{0} 1$ have the same homoclinic braid type. That is,

Lemma 3.9. Let $w$ be a decoration. If $w \neq \star$, then the four homoclinic orbits represented by $P_{0}^{w}$ all have the same homoclinic braid type. Likewise, the two homoclinic orbits represented by $P_{0}^{\star}$ have the same homoclinic braid type.

It is proved in [de Carvalho and Hall 02a] that for each decoration $w$ and each $q<q_{w}$, all of the (four or fewer) periodic orbits represented by $P_{q}^{w}$ also have the same braid type.

\subsection{Topological Train Track Types}

Let $P$ be a periodic orbit of the horseshoe of pseudoAnosov braid type. A Bestvina-Handel train track for $P$ is a pair $(G, g)$, where $G$ is a connected finite graph without valence one or two vertices embedded in $D^{2} \backslash P$ and $g: G \rightarrow G$ is a graph map such that

(a) $G$ has a peripheral subgraph $\Pi$, consisting of a loop around each point of $P$, and $g$ restricts to a homeomorphism $\Pi \rightarrow \Pi$. There is exactly one vertex of $G$ on each component of $\Pi$.

(b) The subgraph $T$ of $G$ consisting of edges not in $\Pi$ is a tree. This implies that there exists a retraction $r: D^{2} \backslash P \rightarrow G$. 
(c) $g: G \rightarrow G$ sends vertices to vertices and is homotopic to $\left.r \circ F\right|_{G}: G \rightarrow G$.

(d) $g: G \rightarrow G$ is efficient; that is, every iterate $g^{n}: G \rightarrow$ $G$ is locally injective away from the vertices of $G$.

In [Bestvina and Handel 95], Bestvina and Handel give an algorithm for constructing such a train track for any periodic orbit $P$ of pseudo-Anosov braid type (the condition that each component of $\Pi$ contains only one vertex of $G$ can be ensured when $P$ is a single periodic orbit by starting the algorithm with a graph which satisfies this condition). The pseudo-Anosov homeomorphism $\phi$ in the isotopy class of $F:\left(D^{2}, P\right) \rightarrow\left(D^{2}, P\right)$ is semiconjugate to $g: G \rightarrow G$. Indeed, a Markov partition for $\phi$ can be constructed by thickening up the edges of $G$ into Markov boxes with nonnegative widths and lengths given by the row and column eigenvectors corresponding to the maximal eigenvalue of the transition matrix of $g: G \rightarrow G$, providing a construction of the invariant measured foliations of $\phi$.

This paper is concerned with an equivalence relation on horseshoe periodic orbits of pseudo-Anosov braid type which corresponds to their train tracks being "essentially the same." Since two periodic orbits of different periods have different numbers of loops in the peripheral subgraphs of their train tracks, the first step is to restrict attention to the trees obtained when the edges of the peripheral subgraph are removed.

Let $P$ be a horseshoe periodic orbit of pseudo-Anosov braid type, and let $(G, g)$ be a Bestvina-Handel train track for $P$. The corresponding reduced train track for $P$ is the pair $(T, t)$, where $T$ is the tree embedded in $D^{2} \backslash P$ whose edges are the nonperipheral edges of $G$ and which has no valence two vertices (i.e., any vertices of $G$ which become valence two vertices of $T$ are deleted), and $t: T \rightarrow$ $T$ is the tree map obtained from $g: G \rightarrow G$ by restricting to $T$ and deleting peripheral loops in image edge-paths. Note that $t$ is locally injective away from vertices of $T$ and preimages of points where the peripheral loops of $G$ were attached. An initial edge of $T$ is one whose counterpart in $G$ has one end (its free end) on the peripheral loop surrounding the leftmost point of $P$.

Let $P$ and $Q$ be two horseshoe periodic orbits of pseudo-Anosov braid type. Write $P \triangleright Q$ if $P$ and $Q$ have reduced train tracks $\left(T_{P}, t_{P}\right)$ and $\left(T_{Q}, t_{Q}\right)$, respectively, each with only one initial edge (denoted $e_{P}$ and $e_{Q}$, respectively), such that there is an orientation-preserving homeomorphism $\psi:\left(D^{2}, P\right) \rightarrow\left(D^{2}, Q\right)$ sending $T_{P}$ onto $T_{Q}$, with

(a) $t_{Q}(x)=\psi \circ t_{P} \circ \psi^{-1}(x)$ for all $x \in T_{Q} \backslash e_{Q}$. (b) There is an embedding $\theta: e_{Q} \rightarrow e_{Q}$ sending the nonfree end of $e_{Q}$ to itself such that $t_{Q}(x)=\psi \circ t_{P} \circ$ $\psi^{-1} \circ \theta(x)$ for all $x \in e_{Q}$.

Intuitively, the reduced train track of $Q$ is the same as the reduced train track of $P$, except that the image of its initial edge has been shortened. It is therefore clear that if $P \triangleright Q$, then $P \geq Q$. $P$ and $Q$ are said to have the same topological train track type if either $P \triangleright Q$ or $Q \triangleright P$.

In particular, if $P$ and $Q$ have the same topological train track type, then the invariant foliations of the corresponding pseudo-Anosov homeomorphisms have the same number of interior singularities with each number $v>2$ of prongs, and the prongs are permuted in the same way by the actions of the pseudo-Anosovs.

Note that Bestvina-Handel train tracks, and hence reduced train tracks, are not unique. In particular (cf. the discussion in Section 3.3 of [Bestvina and Handel 95]), the train track graph can have vertices which don't correspond to singularities of the measured foliations, or whose valence is different from the number of prongs at the corresponding singularity. In this paper, train tracks will always be chosen so that this is not the case. Thus the invariant foliations of a pseudo-Anosov homeomorphism $\psi:\left(D^{2}, P\right) \rightarrow\left(D^{2}, P\right)$ with reduced train track $(T, t)$ have a one-pronged singularity at each point of $P$; an interior $v$-pronged singularity for each vertex of $T$ of valence $v \geq 3$; and a boundary singularity at which the number of prongs depends on the period of $P$. Such train tracks can always be found (see [Franks and Misiurewicz 93], for example).

A convenient notation for describing a reduced train track $(T, t)$ is to start at the free end of the initial edge of $T$ and move around $T$ in the positive direction, numbering edges sequentially as they are encountered and listing each edge in the order in which it is encountered; and then listing in turn the image edge paths. Thus, for example, for the topological train track type corresponding to $w=1$ in Table $1, T$ would be described by the list 1223445531 , and $t$ by $(1223,4,553,1,2)$. A program which takes as input a decoration, and returns the corresponding topological train track type in this format, can be found at [Hall 02]. Note that, in constrast to standard Markov partition conventions, the image of the initial edge need only intersect the interior of, rather than cover, the first edge in its image edge-path. If $P$ and $Q$ have the same topological train track type (say $P \triangleright Q$ ), then the descriptions of the trees $T_{P}$ and $T_{Q}$ are equal, while those of the tree maps $t_{P}$ and $t_{Q}$ differ only in that 
the image edge path of the initial edge of $Q$ is obtained from that of $P$ by deleting $i \geq 0$ initial symbols.

\subsection{Statement of the Conjecture}

Given two decorations $w_{1}$ and $w_{2}$, write $w_{1} \sim w_{2}$ if $\operatorname{hbt}\left(P_{0}^{w_{1}}\right)=\operatorname{hbt}\left(P_{0}^{w_{2}}\right)$; and write $w_{1} \succeq w_{2}$ if $\operatorname{hbt}\left(P_{0}^{w_{1}}\right) \geq \operatorname{hbt}\left(P_{0}^{w_{2}}\right)$ (notice that $\operatorname{hbt}\left(P_{0}^{w}\right)$ is welldefined by Lemma 3.9). Recall that $\mathcal{D}_{w}$ denotes the set of all periodic and homoclinic orbits which have decoration $w$ and that in the notation $P_{q}^{w}$ it is assumed that the height $q$ is compatible with the decoration $w$ (i.e. $\left.q<=q_{w}\right)$.

Conjecture 3.10. Let $w$ and $w^{\prime}$ be decorations. Then

(i) $\operatorname{bt}\left(P_{q}^{w}\right)=\operatorname{bt}\left(P_{q^{\prime}}^{w^{\prime}}\right)$ if and only if $q=q^{\prime}$ and $w \sim w^{\prime}$.

(ii) If $0<q<q_{w}$, then $P_{q}^{w}$ has pseudo-Anosov braid type.

(iii) All of the periodic orbits in $\left\{P_{q}^{w}: 0<q<q_{w}\right\}$ have the same topological train track type.

(iv) The family $\mathcal{D}_{w}$ is totally ordered by $\leq$, with $P_{q}^{w} \leq$ $P_{r}^{w}$ if and only if $q \geq r$.

(v) If $q<q^{\prime}$ and $w \succeq w^{\prime}$, then $P_{q}^{w} \geq P_{q^{\prime}}^{w^{\prime}}$.

The five parts of this conjecture have each been stated in full without common hypotheses because there are different types and amounts of evidence for the different statements, and it is therefore desirable to be able to treat them separately. Notice in particular that part (v) implies part (iv), that part (iii) depends on part (ii), and that if part (i) is false, then the statements of the other parts would have to be changed to reflect this.

Conjecture 3.10 could also be stated for periodic orbits only, leaving out the homoclinic orbits $P_{0}^{w}$. The reason for their inclusion is that they appear naturally when the problem is considered in terms of pruning theory (Section 4.3), which was the original motivation for the conjecture.

The conjecture addresses two problems: if $P$ and $Q$ are horseshoe periodic orbits, is it true (a) that $\operatorname{bt}(P)=$ bt $(Q)$, and (b) that $P \leq Q$ ? (Being able to answer (b) in general provides an answer to (a), since bt $(P)=\operatorname{bt}(Q)$ if and only if $P \leq Q$ and $Q \leq P$.) It does this by rephrasing them in terms of the same questions for homoclinic orbits, which are not, in general, any easier to answer than the original question. Nevertheless, if the conjecture can be proved, it will add considerably to our understanding of the problem in two distinct ways:

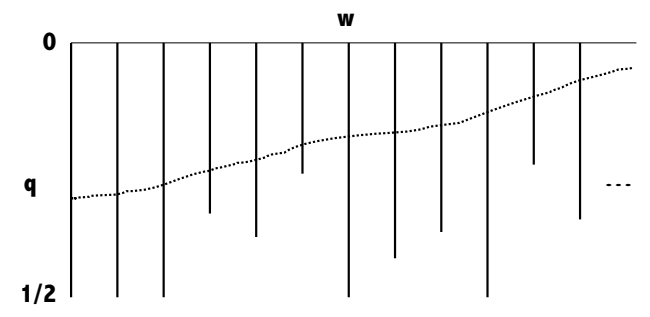

FIGURE 2. A schematic illustration of the conjecture.

1. On the theoretical level, it provides a better understanding of the global structure of $(\mathrm{HS}, \leq)$ : the partial order has been "factored" into a total order (within families with fixed decoration) and the partial order on decorations. Figure 2 provides a schematic illustration: each periodic orbit of the horseshoe is parameterised by its height $q$ and decoration $w$. Each family of orbits with a given equivalence class of decorations is represented by a vertical line: the orbits in such a family are created monotonically from bottom to top as a horseshoe is created. The complication in (HS, $\leq$ ) has been shuffled away in this figure by imagining that $\preceq$ is a total order (perhaps we have restricted to those periodic orbits corresponding to a given chain of decorations) - it is assumed that $w_{1} \succeq w_{2}$ whenever $w_{1}$ is to the left of $w_{2}$. Thus progress through a given family to height $q$ implies progress through all families to its right to at least height $q$, and hence a partially formed horseshoe can be represented by an upward sloping line through the families (as depicted in the figure).

2. On a practical level, showing that two periodic orbits $P_{q}^{w_{1}}$ and $P_{q}^{w_{2}}$ of relatively small period have the same braid type implies that $P_{q^{\prime}}^{w_{1}}$ and $P_{q^{\prime}}^{w_{2}}$ have the same braid type for all $0<q, q^{\prime}<=q_{w_{1}}=q_{w_{2}}$. For example, it can be shown directly (by constructing a conjugacy between the corresponding braids) that the periodic orbits $P_{1 / 3}^{10}$ and $P_{1 / 3}^{01}$ with codes 10011101 and 10011011 have the same braid type. It follows from the conjecture that the orbits with codes $c_{q 1}{ }^{0} 10_{1}^{0}$ and $c_{q 1}^{0} 01_{1}^{0}$ have the same braid type for all $q$ with $0<q \leq q_{10}=1 / 3$.

Under the assumption that part (iii) of the conjecture is true, Table 1 shows the topological train track types (including images) corresponding to decorations of lengths 3 or less. Decorations which are equivalent under $\sim$ are shown in the same row. The train tracks have been 


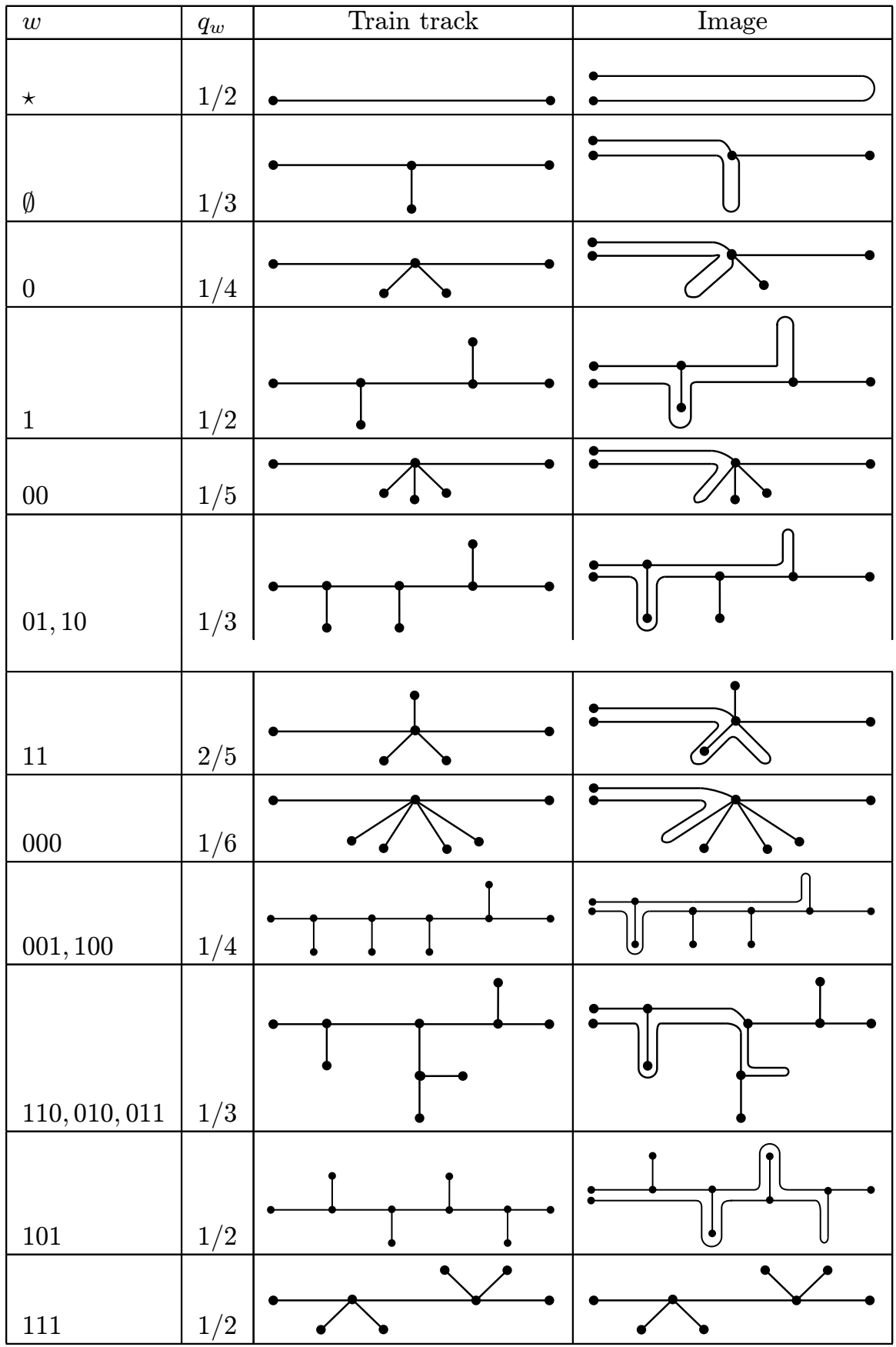

TABLE 1. Topological train track types for short decorations.

drawn in such a way that the free endpoint of the initial edge and its preimage are connected by a horizontal line.

The question, posed in Section 3.2, of whether there exists a horseshoe orbit $P$ whose braid type is distinct from that of $\widehat{P}$ is, under the assumption that part (i) of the conjecture is true, very closely related to the question of whether there exists a decoration $w$ which is not $\sim$ equivalent to its reverse $\widehat{w}$. To see why this is so, note first that $q_{w}=q_{\widehat{w}}$ for all decorations $w$. By Lemma 3.3, $q_{w}$ is the height of the periodic orbit containing the point with itinerary $\overline{10 w 0}$, and as stated in Section 3.2, this is the same as the height of the orbit containing the reverse itinerary $\overline{0 \widehat{w} 01}$ : applying Lemma 3.3 again, this is equal to $q_{\widehat{w}}$. Now let $P=P_{q}^{w}$ be any horseshoe orbit with $q<q_{w}$. Then $P$ has code $c_{q 1}^{0} w_{1}^{0}$, and so $\widehat{P}$ contains the point with itinerary ${ }_{1}^{0} \widehat{w}_{1}^{0} \widehat{c_{q}}$. Since $c_{q}$ is palindromic and 
$q<q_{w}=q_{\widehat{w}}, \widehat{P}$ has code $c_{q}{ }_{1}^{0} \widehat{w}_{1}^{0}$, i.e., $\widehat{P}=P_{q}^{\widehat{w}}$. Hence $P$ and $\widehat{P}$ have the same braid type if and only if $w \sim \widehat{w}$.

\section{EVIDENCE FOR THE CONJECTURE}

The current evidence for the conjecture is of three types: proofs of some special cases, computational evidence for low period orbits with short decorations, and an intuitive justification using pruning theory [de Carvalho 99, de Carvalho and Hall 01].

\subsection{Proofs of Special Cases}

The simplest case, in which $w=\star$, was treated in [Hall 94a], where it was shown that parts (i), (ii), (iii), and (iv) of the conjecture hold for this decoration. (Note that, since $\star$ is not equivalent to any other decoration, in this case part (i) says that the pair of periodic orbits $P_{q}^{\star}$ are the only horseshoe periodic orbits of their braid type for each $q \in \mathbb{Q} \cap(0,1 / 2)$.) Using the techniques of this paper, it doesn't seem hard to show that parts (ii), (iii), and (iv) of the conjecture hold for any given decoration $w$, simply by calculating the appropriate isotopy classes and showing that they leave invariant train tracks of the given type. For example, parts (ii), (iii), and (iv) are proved in [Hall 94b] for decorations of the form $w=1^{2 i-1}$, where $i \geq 1$ is an integer: it is also shown there that for each $q<q_{w}=1 / 2$, the four orbits $P_{q}^{1^{2 i-1}}$ are the only horseshoe orbits of their braid type (the topological train track types which arise are discussed later in this section). However, treating individual cases in this way seems a rather pointless endeavour; the challenge in proving the conjecture is to develop techniques which are applicable when no information about topological train track type is available.

Part (v) of the conjecture only makes sense when a family of decorations is considered simultaneously. In [de Carvalho and Hall 02b], it is shown that all five parts of the conjecture hold when attention is restricted to the set $\left\{w_{q}: q \in(0,1 / 2) \cap \mathbb{Q}\right\}$ of decorations, where $w_{q}$ is the word obtained from $c_{q}$ by deleting the initial symbols 10 and the final symbols 01 (it satisfies $q_{w_{q}}=q$ ). In this case, the forcing order can be simply expressed:

$$
P_{r}^{w_{q}} \geq P_{r^{\prime}}^{w_{q^{\prime}}} \quad \text { if and only if } \quad[r, q] \supseteq\left[r^{\prime}, q^{\prime}\right] .
$$

The conjecture says nothing about the particular topological train track types corresponding to particular decorations. However, studying Table 1 and its extensions to longer decorations makes it very tempting to produce conjectures, the simpler of which seem relatively easy to prove by brute force calculations of the action of the appropriate isotopy classes on trial train tracks. Thus, for example, in [Hall 94b] it is shown that if $w=1^{2 i-i}$ for some integer $i \geq 1$, then the reduced train track has a single period 2 orbit of valence $i+2$ vertices (see $w=1$ and $w=111$ in Table 1$)$. That is,

$$
\begin{array}{r}
T=12233 \ldots(i+1)(i+1)(i+2)(i+3)(i+3) \\
(i+4)(i+4) \ldots(2 i+3)(2 i+3)(i+2) 1,
\end{array}
$$

and

$$
\begin{aligned}
t=( & 12233 \ldots(i+1)(i+1)(i+2),(i+3), \\
& (i+4), \ldots,(2 i+2),(2 i+3)(2 i+3) \\
& (i+2), 1,2,3, \ldots,(i+1)) .
\end{aligned}
$$

Likewise in [de Carvalho and Hall 02b], it is shown that if $q=m / n \in(0,1 / 2)$, and the length $n-3$ decoration $w_{q}$ is obtained from $c_{q}$ by deleting the initial 10 and the final 01 , then $T$ has a single fixed valence $n$ vertex, and the action of $t$ on noninitial edges of $T$ is rotation by $m / n$ (see $w=\emptyset, 0,00,11$ and 000 in Table 1, corresponding to $q=1 / 3,1 / 4,1 / 5,2 / 5$, and $1 / 6$, respectively).

Examining the topological train track types corresponding to $w=1,10$, and 100 also leads to an obvious conjecture about the case $w=10^{n}$, which can be proved with a lot of work but not much difficulty. A more interesting conjecture, however, is that if $P$ is a period $n$ horseshoe orbit with code $c_{P}$, and if $w$ is obtained from $c_{P}$ by deleting the final symbol, then the corresponding topological train track type has a single period $n$ orbit of valence 3 vertices, and the braid type of this periodic orbit is that of $P$. This conjecture remains unproved.

\subsection{Computational Evidence}

The main tool which the authors have used for computational investigation of Conjecture 3.10 is an implementation by the first author of the Bestvina-Handel train track algorithm. Using this, parts (ii), (iii), and (iv) of the conjecture have been checked directly for all periodic orbits whose decoration is of length 8 or less, and whose height has denominator 10 or less. Specifically, it was verified that for each such decoration $w$,

(a) All of the orbits $P_{q}^{w}(0)$ and $P_{q}^{w}(1)$ with $q<q_{w}$ have pseudo-Anosov braid type and the same topological train track type.

(b) The pseudo-Anosov representatives of the orbits $P_{q}^{w}(0)$ and $P_{q}^{w}(1)$ have the same topological entropy for each $q<q_{w}$. 


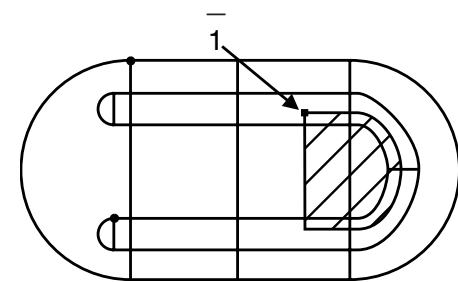

(a)

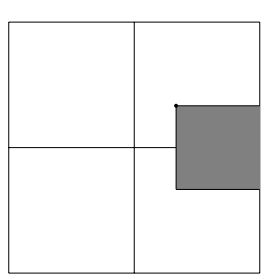

(b)

FIGURE 3. A pruning disk in the horseshoe.

(c) For each $q_{1}<q_{2}<q_{w}$, the pseudo-Anosov representative of the orbit $P_{q_{1}}^{w}(0)$ has greater topological entropy than that of the orbit $P_{q_{2}}^{w}(0)$.

This check involved calculating train tracks for 15566 periodic orbits. Naturally there is no constraint other than time preventing the continuation of this search; the program used is available on the web [Hall 02]. Part (i) is much harder to check computationally however, since determining whether or not two decorations $w$ and $w^{\prime}$ are equivalent is essentially the same problem as the conjugacy problem in the braid group, and as such becomes impractical for quite short decorations. The authors do not have any good computational approach to part (v) of the conjecture.

\subsection{Pruning Theory}

Pruning is a mechanism for destroying dynamics of surface homeomorphisms in a controlled manner. The following account avoids some technical details: a full description can be found in [de Carvalho 99, de Carvalho and Hall 01].

Let $F: D^{2} \rightarrow D^{2}$ be a homeomorphism (which here will always be the horseshoe). A pruning region for $F$ is an $F$-invariant open set $\mathcal{R}$ such that there exists an isotopy supported in $\mathcal{R}$ which destroys the dynamics there; that is, if $F_{\mathcal{R}}$ denotes the homeomorphism obtained at the end of the isotopy, then every point of $\mathcal{R}$ is wandering under $F_{\mathcal{R}}$. Since the isotopy is supported in $\mathcal{R}, F_{\mathcal{R}}$ is equal to $F$ outside $\mathcal{R}$.

Example 4.1. Consider the disk shown in Figure 3 (a), which is bounded by segments of the stable and unstable manifolds of the fixed point of the horseshoe with code 1 . Then $\mathcal{R}=\bigcup_{n \in \mathbb{Z}} F^{n}(\operatorname{Int}(D))$ is a pruning region for $F$ (see [de Carvalho 99] for the construction of the isotopy in this case).
As in this example, pruning regions are usually presented as saturations of open sets under the dynamics. Such a generating open set is called a pruning front, and these will be used to describe pruning regions in what follows.

Pruning fronts are themselves unions of pruning disks which are, roughly speaking, open disks bounded by segments of the stable and unstable manifolds of (possibly different) periodic points, as in the example above. The figures shown in the remainder of this section all contain a square, which represents the nonwandering set of the horseshoe (after collapsing gaps in the Cantor set). Pruning disks will be depicted with shaded boxes: together, they make up the pruning front, which in turn yields the pruning region under saturation. The schematic representation of the pruning disk in the above example is shown in Figure 3 (b).

A general pruning disk can be specified by the horizontal and vertical coordinates of its edges. The simplest type of pruning disk is a vertical pruning disk, which extends all the way from the bottom to the top of the square, and from some point up to the right of the square. A vertical pruning disk can be specified by the horizontal coordinate of its left edge, which will be referred to as its horizontal coordinate.

In [de Carvalho and Hall 01], the Bestvina-Handel algorithm is recast in the language of pruning to give an alternative algorithmic proof of Thurston's classification theorem for surface homeomorphisms. Given a periodic orbit $P$ (or indeed any finite invariant set) of the horseshoe, this algorithm yields a maximal pruning region $\mathcal{R}=\mathcal{R}(P)$ for $F$ relative to $P$ with the property that, after collapsing wandering domains, $F_{\mathcal{R}}$ is the Thurston representative in the isotopy class of $F:\left(D^{2}, P\right) \rightarrow\left(D^{2}, P\right)$. The pruning region is maximal relative to $P$ in the sense that no further dynamics can be destroyed by pruning without destroying the periodic orbit $P$ itself. The same algorithm appears to work for 
homoclinic orbits of the horseshoe. A proof that this is the case might be obtained by combining the techniques of [de Carvalho and Hall 01] with those of [Hulme 00].

Note that maximal pruning regions are not unique. The reason is that there are different regions of the dynamics of the horseshoe which are conjugate to each other; in carrying out the algorithm, one has to decide which of two conjugate regions should be destroyed. Different choices lead to homeomorphisms $F_{\mathcal{R}_{1}}$ and $F_{\mathcal{R}_{2}}$ which, though conjugate, are described by different pruning regions. This phenomenon will be illustrated in Example 4.3 (d). This nonuniqueness is probably the main cause of difficulty in proving the conjectures in this paper.

The following conjecture, if proved, would provide the main technical ingredient for proving parts (iv) and (v) of Conjecture 3.10. It uses the notation $\mathcal{D}_{w}^{s}$ to denote the family of orbits $\left\{P_{q}^{w}(s): 0<q<=q_{w}\right\}$, where $s \in\{0,1\}$.

Conjecture 4.2. Let $w$ and $w^{\prime}$ be decorations with $w \succeq w^{\prime}$, and consider (any particular choices of) the homoclinic orbits $P_{0}^{w}$ and $P_{0}^{w^{\prime}}$. Then there exist $s, s^{\prime} \in\{0,1\}$ and maximal pruning regions $\mathcal{R}_{w}$ and $\mathcal{R}_{w^{\prime}}$ for $F$ relative to $P_{0}^{w}$ and $P_{0}^{w^{\prime}}$ respectively, such that:

(i) $\mathcal{R}_{w^{\prime}}$ is disjoint from $\mathcal{D}_{w^{\prime}}^{s^{\prime}}$ and $\mathcal{R}_{w}$ is disjoint from both $\mathcal{D}_{w}^{s}$ and $\mathcal{D}_{w^{\prime}}^{s^{\prime}}$.

(ii) Let $q<q_{w}$ and $q^{\prime}<q_{w^{\prime}}$. Then a maximal pruning region for the periodic orbit $P_{q}^{w}(s)$ (respectively $P_{q^{\prime}}^{w^{\prime}}\left(s^{\prime}\right)$ ) can be obtained by adding to $\mathcal{R}_{w}$ (respectively $\left.\mathcal{R}_{w^{\prime}}\right)$ the saturation of the vertical pruning disk with horizontal coordinate $\overline{c_{q} s w_{1}^{0}}$ (respectively $\left.\overline{c_{q^{\prime}} s^{\prime} w_{1}^{\prime 0}}\right)$.

These statements could be rephrased in terms of equivalent decorations. For example, in (i) above, instead of saying that it is possible to find $\mathcal{R}_{w}$ disjoint from $\mathcal{D}_{w}^{s}$, it could be said that given any maximal pruning region $\mathcal{R}_{w}$ for $\mathrm{F}$ relative to $P_{0}^{w}$, there exists a decoration $v \sim w$ and $s \in\{0,1\}$ such that $\mathcal{R}_{w}$ is disjoint from all orbits in the family $\mathcal{D}_{v}^{s}$. The point is that a maximal pruning relative to $P_{0}^{w}$ may destroy some orbits of the relevant braid types, but cannot destroy all of them.

If Conjecture 4.2 holds, then Conjecture 3.10 (v) follows (and hence so also does Conjecture 3.10 (iv)). For suppose $q<q^{\prime}$ and $w \succeq w^{\prime}$, and let $\mathcal{R}_{w}, \mathcal{R}_{w^{\prime}}, s$, and $s^{\prime}$ be as given by Conjecture 4.2. The fact that $q<q^{\prime}$ implies that $\overline{c_{q} s w_{1}^{0}}$ is greater than $\overline{c_{q^{\prime}} s^{\prime} w_{1}^{\prime 0}}$ in the unimodal order, and hence that the vertical pruning disk $\mathcal{V}$ with horizontal coordinate $\overline{c_{q} s w_{1}^{0}}$ contains no point in the periodic orbit $P_{q^{\prime}}^{w^{\prime}}\left(s^{\prime}\right)$. By part (i) of the conjecture $\mathcal{R}_{w}$ is also disjoint from $P_{q^{\prime}}^{w^{\prime}}\left(s^{\prime}\right)$, and hence by part (ii), there is a maximal pruning region for $P_{q}^{w}(s)$ which is disjoint from $P_{q^{\prime}}^{w^{\prime}}\left(s^{\prime}\right)$. Thus the pseudo-Anosov representative of the braid type of $P_{q}^{w}(s)$ contains a periodic orbit of the braid type of $P_{q^{\prime}}^{w^{\prime}}\left(s^{\prime}\right)$, that is, $P_{q}^{w} \geq P_{q^{\prime}}^{w^{\prime}}$.

The motivation for this conjecture is again computational. It seems very natural once one has calculated maximal pruning regions for homoclinic and periodic orbits with many different decorations. The following examples are illustrative of such computations.

\section{Example 4.3.}

(a) Let $w=\emptyset$. Figure 4 (a) depicts the homoclinic orbit $P_{0}^{\emptyset}$ (with core 1001), together with a maximal pruning front $\mathcal{F}^{\emptyset}$ (which generates $\mathcal{R}_{\emptyset}$ under saturation). In Figure 4 (b), the periodic orbits $P_{1 / 4}^{\emptyset}$ and $P_{2 / 7}^{\emptyset}$ (with codes 1000100 and 1001100100) are shown (depicted $\bullet$ and $\circ$ respectively), together with a maximal pruning front $\mathcal{F}_{1 / 4}^{\emptyset}$ relative to $P_{1 / 4}^{\emptyset}$, which is precisely $\mathcal{F}^{\emptyset}$ together with a vertical pruning disk with horizontal coordinate $\overline{1000100}$. Since $2 / 7>1 / 4$, the rightmost point of $P_{2 / 7}^{\emptyset}$ lies to the left of this vertical pruning disk, and hence no point of $P_{2 / 7}^{\emptyset}$ falls into $\mathcal{F}_{1 / 4}^{\emptyset}$. It follows that $P_{1 / 4}^{\emptyset}$ forces $P_{2 / 7}^{\emptyset}$.

The fixed point of code 1 is also depicted (with $\square$ ) in Figure 4 (a). The fact that $\mathcal{F}^{\emptyset}$ has this fixed point on its boundary accounts for the topological train track type corresponding to the decoration $\emptyset$ having a fixed valence 3 vertex (see Table 1).

(b) A similar treatment of the decoration $w=110$ yields Figure 5. In (a) the homoclinic orbit $P_{0}^{110}$ with core 1011001 is depicted together with a maximal pruning front $\mathcal{F}^{110}$. Note that in this case the maximal pruning front consists of two pruning disks. The fixed point of code 1 and the period 3 orbit of code 100 are also shown. The fact that they lie on the boundary of $\mathcal{F}^{110}$ accounts for the topological train track type corresponding to the decoration 110 having four valence three vertices, one fixed, and the others lying on a period 3 orbit.

In Figure 5 (b), a maximal pruning front $\mathcal{F}_{1 / 4}^{110}$ relative to the periodic orbit $P_{1 / 4}^{110}$ with code 1000101100 is shown; it is exactly $\mathcal{F}^{110}$ together with a vertical pruning disk with horizontal coordinate $\overline{1000101100}$. 


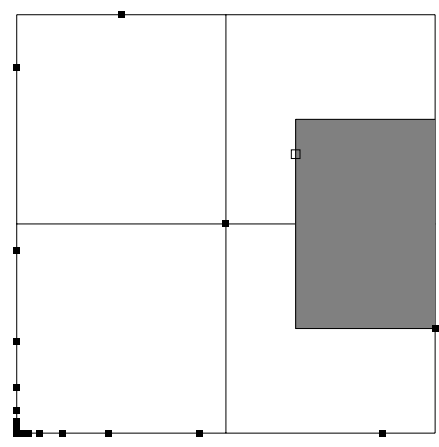

(a)

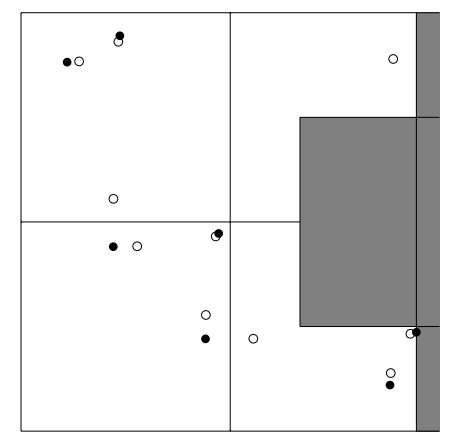

(b)

FIGURE 4. Maximal pruning fronts for the decoration $\emptyset$.

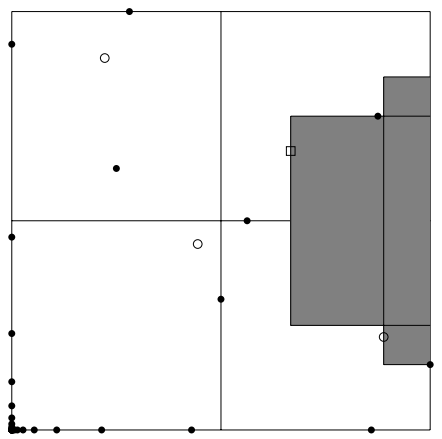

(a)

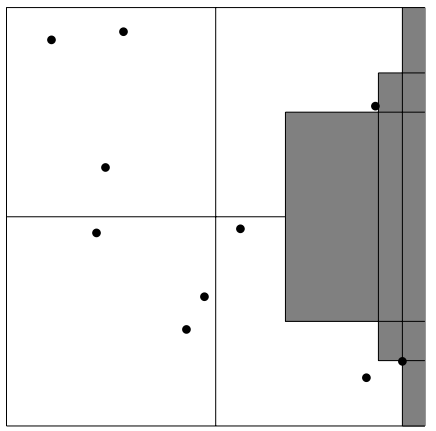

(b)

FIGURE 5. Maximal pruning fronts for the decoration 110.

(c) This example illustrates part (v) of Conjecture 3.10. Let $w=1$. Figure 6 (a) depicts the homoclinic orbit $P_{0}^{1}$ (with core 10101, shown as $\bullet$ ), together with a maximal pruning front $\mathcal{F}^{1}$. The homoclinic orbit $P_{0}^{\emptyset}$ is also shown (o); since it lies outside $\mathcal{F}^{1}$, it follows that the decoration 1 forces the empty decoration, i.e., $1 \succ \emptyset$. In Figure 6 (b), the periodic orbits $P_{1 / 4}^{1}(1)$ and $P_{2 / 7}^{\emptyset}(0)$ (with codes 10001111 and 1001100100, respectively) are shown (depicted $\bullet$ and $\circ$, respectively), together with a maximal pruning front $\mathcal{F}_{1 / 4}^{1}$ relative to $P_{1 / 4}^{1}$, which is precisely $\mathcal{F}^{1}$ together with a vertical pruning disk with horizontal coordinate $\overline{10001111}$. Since $2 / 7>1 / 4$, the orbit $P_{2 / 7}^{\emptyset}$ is disjoint from this vertical pruning disk; and since $1 \succ \emptyset$, it was possible to choose $\mathcal{F}^{1}$ to be disjoint from $\mathcal{D}_{\emptyset}^{0}$. Thus $P_{2 / 7}^{\emptyset}$ is disjoint from $\mathcal{F}_{1 / 4}^{1}$, i.e. $P_{1 / 4}^{1} \geq P_{2 / 7}^{\emptyset}$.

(d) The final example illustrates the nonuniqueness of maximal pruning fronts. Let $w=010$. As noted in Table 1, the three decorations 010,110 , and 011 are all equivalent, i.e., $010 \sim 110 \sim 011$. The simplest maximal pruning front relative to the homoclinic orbit $H=P_{0}^{010}$ consists of two pruning disks, and is depicted in Figure 7. $H$ itself is not depicted in this figure: instead, the periodic orbit $P_{1 / 5}^{010}(0)$ is shown. It can be seen that this periodic orbit lies in the pruning region, and hence is destroyed during the pruning isotopy. (In fact the same is true for all of the periodic orbits in $\mathcal{D}_{010}$.)

There are two ways to resolve this problem. The first, following the original formulation of Conjecture 4.2 , is to find a different maximal pruning front which is disjoint from the periodic orbits in $\mathcal{D}_{010}^{0}$. Such a maximal pruning front exists, but it consists of infinitely many pruning disks. The second, following the restatement of Conjecture 4.2, is to observe that the maximal pruning front of Figure 7 avoids the orbits of the family $\mathcal{D}_{110}^{0}$ (although it meets those of $\left.\mathcal{D}_{110}^{1}\right)$. Although this is a much simpler resolution in this particular case, it does not appear to be a practical approach in general, since computation of the equivalence relation $\sim$ on decorations seems to be quite intractable. 


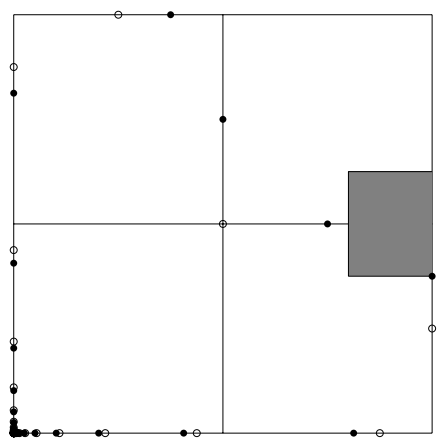

(a)

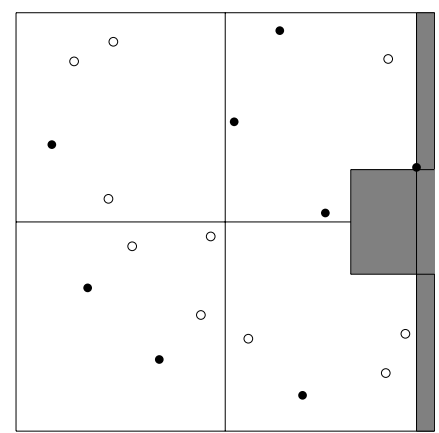

(b)

FIGURE 6. Maximal pruning fronts for the decoration 1.

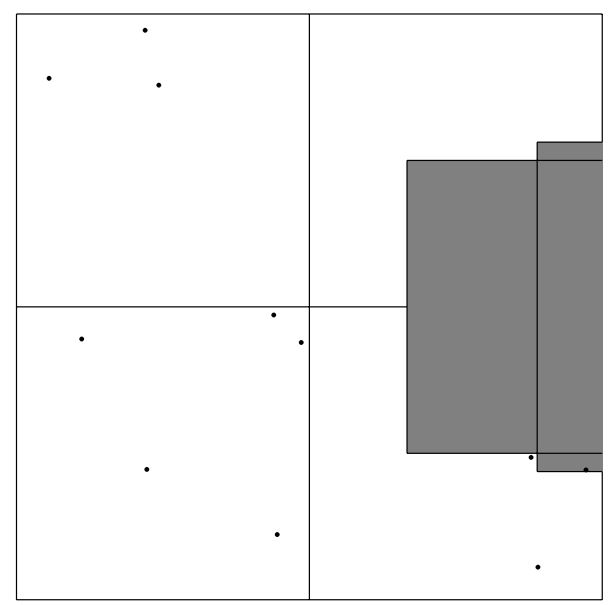

FIGURE 7. Maximal pruning front for the decoration 010.

The nonuniqueness of maximal pruning regions, as shown in Example 4.3(d), is a major obstacle to proving the conjecture. On the one hand, there are some natural choices for a maximal pruning region relative to $P_{0}^{w}$ as one follows the algorithm described in [de Carvalho and Hall 01]. However these natural pruning regions sometimes contain the family $\mathcal{D}_{w}$. If, on the other hand, one tries to obtain a maximal pruning region which avoids this family, the construction may require infinitely many steps, and may indeed cease to be algorithmic. The alternative approach, to find an equivalent family $\mathcal{D}_{v}$ which is untouched by the natural maximal pruning region, would require a far deeper understanding of the relation $\sim$ than seems possible at present.

\section{APPENDIX: PROOF OF LEMMA 3.3}

The following technical lemma from [de Carvalho and Hall $02 b]$ is required in the proof.
Lemma 5.1. Let $0<m / n<1 / 2$, and write $c_{m / n}=$ $10^{\kappa_{1}} 1^{2} 0^{\kappa_{2}} 1^{2} \ldots 1^{2} 0^{\kappa_{m}} 1$. Let $1 \leq r \leq m$. Then the word

$$
c=10^{\kappa_{r}+1} 1^{2} 0^{\kappa_{r+1}} 1^{2} 0^{\kappa_{r+2}} 1^{2} \ldots 1^{2} 0^{\kappa_{m}} 1
$$

disagrees with $c_{m / n}$ within the shorter of their lengths, and is greater than it in the unimodal order.

The next observation, which follows immediately from the fact that the height algorithm terminates at an isolated 1, is used several times in the proof: it is therefore stated as a lemma for ease of reference.

Lemma 5.2. Let $c \in\{0,1\}^{\mathbb{N}}$ contain the word 010 , and let $v$ be the shortest initial subword of $c$ which ends 010 . Then any $c^{\prime} \in\{0,1\}^{\mathbb{N}}$ which has $v$ as an initial subword satisfies $q\left(c^{\prime}\right)=q(c)$.

It is also necessary in the proof of Lemma 3.3 to extend the definition of height to elements $c \in\{0,1\}^{\mathbb{N}}$ which 
do not contain the word 010 . The following definition (see [Hall 94a] for motivation) can be used:

$$
q(c)=\inf \left\{q \in(0,1 / 2] \cap \mathbb{Q}: q=1 / 2 \text { or } \overline{c_{q} 0} \prec c\right\},
$$

where $\prec$ is the unimodal order on $\{0,1\}^{\mathbb{N}}$. With this definition, $q:\{0,1\}^{\mathbb{N}} \rightarrow[0,1 / 2]$ is a decreasing function which agrees with the previous definition of height on the subset $\mathcal{C}$ of $\{0,1\}^{\mathbb{N}}$.

The final idea which is required in the proof is that of paired periodic orbits of the horseshoe. If $c \in\{0,1\}^{n}$ for some $n$, write $\tilde{c}$ for the element of $\{0,1\}^{n}$ obtained by changing the final symbol of $c$. It is well known (see [Hall 94a] for more details) that for every horseshoe periodic orbit $P$, exactly one of the words $c_{P} \tilde{c}_{P}$ and $\tilde{c}_{P} c_{P}$ is the code of a periodic orbit. Denote this orbit $\mathcal{D}(P)$, the doubling of $P$-its (reducible) braid type is that obtained when a periodic orbit of the braid type of $P$ undergoes a period-doubling bifurcation. From this observation and the fact that $q(P)$ is the lefthand endpoint of the rotation interval of $P$ (or arguing directly with the symbolics), it follows that $q(P)=q(\mathcal{D}(P))$. Moreover, given a horseshoe orbit $Q$, the word $\tilde{c}_{Q}$ is not the code of a periodic orbit of the same braid type as $Q$ if and only if $Q=\mathcal{D}(P)$ for some $P$. In this case, $Q$ is said to be unpaired; otherwise it is paired with the orbit of code $\tilde{c}_{Q}$.

The idea of the proof of Lemma 3.3 is as follows: the case $c_{q} 0 w 0$ is relatively straightforward: the words of the form 010 at the start and end of $0 c_{q} 0$ act as 'stops' for the height algorithm, making it possible to apply Lemma 5.2 to argue directly from the definition of $q_{w}$. Provided $c_{q} 0 w 0$ is paired, the result for $c_{q} 0 w 1$ follows immediately: in the unpaired case, the form of the code of a paired orbit makes it possible to relate $w$ to a shorter decoration, and hence to argue inductively on the length of the decoration. Since $c_{q}$ is palindromic, reversing $\overline{c_{q} 0 \widehat{w} 1}$ gives a shift of $\overline{c_{q} 1 w 0}$, and the result can be established in this case using the reversal properties of horseshoe itineraries discussed in Section 3.2. Finally, the result can be extended to $c_{q} 1 w 1$ by again making use of pairing arguments.

Proof of Lemma 3.3. The lemma can be checked directly when $w=\star$ or $w=\emptyset$, so it will be assumed in the remainder of the proof that $w$ has length $k \geq 1$. The proof is by induction on $k$.

(i) It will be shown first that the code $c_{q} 0 w 0$ is maximal of height $q$ when $q<q_{w}$, and is not maximal of height $q$ when $q>q_{w}$. Suppose first that $q>q_{w}$, and let $0 \leq i \leq k+2$ be an integer such that $q\left(\sigma^{i}(\overline{10 w 0})\right)=q_{w}$. Let $v$ be the shortest initial subword of $\sigma^{i}(\overline{10 w 0})$ which ends 010 . Then $v$ is also a subword of $\overline{c_{q} 0 w 0}$, and hence by Lemma 5.2, there is some $j$ such that $q\left(\sigma^{j}\left(\overline{c_{q} 0 w 0}\right)\right)=q_{w}<q$. Since $q:\{0,1\}^{\mathbb{N}} \rightarrow[0,1 / 2]$ is decreasing, it follows that $c_{q} 0 w 0$ is not maximal.

Next suppose that $q=m / n<q_{w}$, and let $s_{i}=$ $\sigma^{i}\left(\overline{c_{q} 0 w 0}\right)$ for $0<i<k+n+3$. Then $c_{q} 0 w 0$ is maximal if and only if $\overline{c_{q} 0 w 0} \succ s_{i}$ for each $i$. Now the $1 \mathrm{~s}$ at the beginning and the end of $c_{q}$ are both isolated in $\overline{c_{q} 0 w 0}$, while the other $1 \mathrm{~s}$ in $c_{q}$ are not isolated. It follows that if $i<n+1$, then $s_{i}$ disagrees with $c_{q} 0 w 0$ in the first $n+1-i$ symbols, and so $s_{i} \prec \overline{c_{q} 0 w 0}$ (this follows from the maximality of $\overline{c_{q} 0}$ ). On the other hand, if $i \geq n+1$, then $q\left(s_{i}\right) \geq q_{w}>$ $q=q\left(\overline{c_{q} 0 w 0}\right)$ by Lemma 5.2, and so $s_{i} \prec \overline{c_{q} 0 w 0}$. Hence $c_{q} 0 w 0$ is maximal. Since the height algorithm for $c_{q} 0 w 0$ terminates at the isolated 1 at the end of $c_{q}$, it has height $q$.

(ii) Now consider the code $c_{q} 0 w 1$, and suppose first that $q<q_{w}$. Then $c_{q} 0 w 0$ is maximal of height $q$ by i), and hence the same must be true of $c_{q} 0 w 1$, provided only that $c_{q} 0 w 0$ is a paired orbit. However, if $c_{q} 0 w 0$ is unpaired, then it is the doubling of some orbit with code $c_{q} 0 v 1$ of height $q$ and decoration $v$. Thus $c_{q} 0 w 0=c_{q} 0 v 1 c_{q} 0 v 0$, and $w=v 1 c_{q} 0 v$. Since $w$ contains the word $c_{q}$, it follows that $q_{w} \leq q$; a contradiction.

Now suppose that $q>q_{w}$. If $c_{q} 0 w 1$ were maximal of height $q$, then it couldn't be a paired orbit, since if it were, then $c_{q} 0 w 0$ would be maximal of height $q$, contradicting (i). Suppose then that $c_{q} 0 w 1$ is maximal, of height $q$, and unpaired; hence it is the doubling of some orbit with code $c_{q} 0 v 0$ of height $q$ and decoration $v$, and $q \leq q_{v}$ by the inductive hypothesis. Thus $c_{q} 0 w 1=c_{q} 0 v 0 c_{q} 0 v 1$, and $w=v 0 c_{q} 0 v$. Let $i$ be such that $q_{w}=q\left(\sigma^{i}\left(\overline{10 v 0 c_{q} 0 v 0}\right)\right)$. Since $q_{w}<q$, the shortest initial word of $\sigma^{i}\left(\overline{10 v 0 c_{q} 0 v 0}\right)$ which ends 010 is also contained in $\overline{10 v 0}$, and hence $q_{v} \leq q_{w}$. This contradicts $q \leq q_{v}$.

(iii) Next consider the orbit with code $c_{q} 1 w 0$. Suppose first that $q<q_{w}$. Since $q_{w}=q_{\widehat{w}}$, it follows by (ii) that $c_{q} 0 \widehat{w} 1$ is maximal of height $q$; since $c_{q}$ is palindromic and $q(R)=q(\widehat{R})$ for any horseshoe periodic orbit $R$, it follows that the periodic orbit which contains the point of itinerary $\overline{c_{q} 1 w 0}$ has height $q$. Hence if $c_{q} 1 w 0$ is not maximal of height $q$, then there is some $i$ with $1 \leq i<n+k+2$ such that $c=\sigma^{i}\left(\overline{c_{q} 1 w 0}\right) \succ \overline{c_{q} 1 w 0}$; and $q(c)=q$. However, $i \leq n+2$, since otherwise the shortest initial 
word $v$ of $c$ which ends 010 would be contained in $\overline{10 w 0}$, giving $q_{w} \leq q$ by Lemma 5.2, contradicting $q<q_{w}$. Similarly, $i=n+2$ is impossible, since then $c=\overline{1 w 0 c_{q}}$, and $\overline{10 w 0} \succ \overline{1 w 0 c_{q}}$. Hence there is some $r$ with $2 \leq r \leq m$ such that

$$
c=10^{\kappa_{r}} 1^{2} 0^{\kappa_{r+1}} 1^{2} \ldots 1^{2} 0^{\kappa_{m}} 1^{2} \overline{w 0 c_{q} 1}
$$

Since $q(c)=q$ and $c \succ \overline{c_{q} 1 w 0}$, it follows that

$$
\begin{gathered}
c=10^{\kappa_{r}} 1^{2} 0^{\kappa_{r+1}} 1^{2} \ldots 1^{2} 0^{\kappa_{m}} \quad 1^{2} \overline{w 0 c_{q} 1} \\
=10^{\kappa_{1}} 1^{2} 0^{\kappa_{2}} 1^{2} \ldots 1^{2} 0^{\kappa_{m-r+1}} 1^{2} 0^{\kappa_{m-r+2}} 1^{2} \\
\ldots 1^{2} 0^{\kappa_{m}} 1 \ldots
\end{gathered}
$$

Thus (observing that the word $0 c_{q}$ starts 010) $w=0^{\kappa_{m-r+2}} 1^{2} \ldots 1^{2} 0^{\kappa_{m}-1} \ldots$, and so

$$
\begin{gathered}
10 w 0=10^{\kappa_{m-r+2}+1} 1^{2} 0^{\kappa_{m-r+3}} 1^{2} 0^{\kappa_{m-r+4}} 1^{2} \\
\ldots 1^{2} 0^{\kappa_{m}} 1 \ldots
\end{gathered}
$$

giving $q(\overline{10 w 0}) \leq q$ by Lemma 5.1 , contradicting $q<q_{w}$. Hence $c_{q} 1 w 0$ is maximal of height $q$ as required.

Suppose on the other hand that $q>q_{w}$. If $c_{q} 1 w 0$ were maximal of height $q$, a similar argument to the above would show that $c_{q} 0 \widehat{w} 1$ would also be maximal of height $q$, contradicting ii) and the fact that $q_{w}=$ $q_{\widehat{w}}$.

(iv) That $c_{q} 1 w 1$ is maximal of height $q$ if $q<q_{w}$, and is not maximal of height $q$ if $q>q_{w}$ follows from (iii) in the same way that (ii) follows from (i).

\section{REFERENCES}

[Adler et al. 65] R. Adler, A. Konheim, and M. McAndrew. "Topological entropy." Trans. Amer. Math. Soc. 114 (1965), 309-319.

[Benardete et al. 93] D. Benardete, M. Gutiérrez, and Z. Nitecki. "A combinatorial approach to reducibility of mapping classes." In Mapping class groups and moduli spaces of Riemann surfaces (Göttingen, 1991/Seattle, WA, 1991), pp. 1-31, Amer. Math. Soc., Providence, RI, 1993.

[Benardete et al. 95] D. Benardete, M. Gutiérrez, and Z. Nitecki. "Braids and the Nielsen-Thurston classification." J. Knot Theory Ramifications 4:4 (1995), 549-618.

[Bestvina and Handel 95] M. Bestvina and M. Handel. "Train-tracks for surface homeomorphisms." Topology 34:1 (1995), 109-140.
[Boyland 84] P. Boyland. emphBraid types and a topological method of proving positive entropy, Preprint, Boston University (the results of this preprint are included in the published paper [Boyland 94]), 1984.

[Boyland 92] P. Boyland, "Rotation sets and monotone periodic orbits for annulus homeomorphisms." Comment. Math. Helv. 2 (1992), 203-213.

[Boyland 94] P. Boyland. "Topological methods in surface dynamics." Topology Appl. 58:3 (1994), 223-298.

[de Carvalho 99] A. de Carvalho. "Pruning fronts and the formation of horseshoes." Ergodic Theory Dynam. Systems 19:4 (1999), 851-894.

[de Carvalho and Hall 02a] A. de Carvalho and T. Hall. "Conjugacies between horseshoe braids." Preprint.

[de Carvalho and Hall 02b] A. de Carvalho and T. Hall, "Star shaped train tracks." Preprint.

[de Carvalho and Hall 01] A. de Carvalho and T. Hall, "Pruning theory and Thurston's classification of surface homeomorphisms." J. European Math. Soc. 3:4 (2001), $287-333$.

[Devaney 89] R. Devaney. An introduction to chaotic dynamical systems, Second ed., Addison-Wesley Publishing Company Advanced Book Program, Redwood City, CA, 1989.

[Fathi et. al. 79] A. Fathi, F. Laudenbach, and V. Poénaru. "Travaux de Thurston sur les surfaces." In Société Mathématique de France, Paris, 1979, Séminaire Orsay.

[Franks and Misiurewicz 93] J. Franks and M. Misiurewicz. "Cycles for disk homeomorphisms and thick trees." in Nielsen theory and dynamical systems (South Hadley, MA, 1992), pp. 69-139, Amer. Math. Soc., Providence, RI, 1993.

[Hall 02] T. Hall. Software available from http://www.liv.ac.uk/ tobyhall/hs/.

[Hall 91] T. Hall. "Unremovable periodic orbits of homeomorphisms." Math. Proc. Cambridge Philos. Soc. 110:3 (1991), 523-531.

[Hall 94a] T. Hall. "The creation of horseshoes." Nonlinearity 7:3 (1994), 861-924.

[Hall 94b] T. Hall. New invariants and entropy bounds for partially formed horseshoes, Centre de Recerca Matemàtica preprint No. 269, November 1994.

[Hulme 00] H. Hulme. Finite and infinite braids: a dynamical systems approach, Ph.D. thesis, University of Liverpool, 2000.

[Holmes and Williams 85] P. Holmes and R. Williams. "Knotted periodic orbits in suspensions of Smale's horseshoe: torus knots and bifurcation sequences." Arch. Rational Mech. Anal. 90:2 (1985), 115-194. 
[Katok 80] A. Katok. "Lyapunov exponents, entropy and periodic orbits for diffeomorphisms." Inst. Hautes Etudes Sci. Publ. Math. 51 (1980), 137-173.

[Los 93] J. Los. "Pseudo-Anosov maps and invariant train tracks in the disc: a finite algorithm." Proc. London Math. Soc. (3) 66:2 (1993), 400-430.
[Smale 67] S. Smale. "Differentiable dynamical systems." Bull. Amer. Math. Soc. 73 (1967), 747-817.

[Thurston 88] W. Thurston. "On the geometry and dynamics of diffeomorphisms of surfaces." Bull. Amer. Math. Soc. (N.S.) 19:2 (1988), 417-431.

André de Carvalho, Institute for Mathematical Sciences, State University of New York at Stony Brook, NY 11794-3660 (andre@math.sunysb.edu)

Toby Hall, Department of Mathematical Sciences, University of Liverpool, Liverpool L69 7ZL, UK (T.Hall@liverpool.ac.uk)

Received April 11, 2001; accepted November 13, 2001. 